\title{
Regulation of Intestinal and Hepatic Apoprotein Synthesis after Chronic Fat and Cholesterol Feeding
}

\author{
M. F. Go, G. Schonfeld, B. Pfleger," T. G. Cole," N. L. Sussman, and D. H. Alpers ${ }^{*}$ \\ ${ }^{\ddagger}$ Gastroenterology Division and ${ }^{*}$ Lipid Research Center, Washington University School of Medicine, St. Louis, Missouri 63110
}

\begin{abstract}
Although diet influences levels of lipoproteins and their corresponding apoproteins, its effects on the molecular regulation of apoprotein synthesis are relatively unknown. Male SpragueDawley rats were fed an atherogenic diet containing cholesterol and propylthiouracil (PTU). Intestinal apo AI and AIV mRNA concentrations were decreased by the atherogenic diet, but apo AI and AIV synthesis was increased in vitro (organ explants) and in vivo (polysome runoff), consistent with regulation at the translational level. In contrast, hepatic apo $\mathbf{E}$ mRNA concentration and synthesis were increased after the atherogenic diet, consistent with pretranslational regulation.

The response to cholesterol feeding for hepatic apo $\mathrm{AI}$ and E showed a third pattern of regulation, in which synthesis increased and mRNA content remained stable or fell, again suggesting translational control, but polysome runoff synthesis was unchanged. The apparent importance of translational regulation in the intestine is consistent with the necessity for the tissue to respond rapidly to changes in intraluminal content.
\end{abstract}

\section{Introduction}

The effects of diet-induced hypercholesterolemia on the levels and distributions of lipoproteins and apoproteins in plasma have been examined in man and in several species of animals. Total cholesterol levels in plasma increase 2- to 10-fold and cholesterol enriched migrating species of VLDL ( $\beta$-VLDL), intermediate density lipoproteins, and an apo E-rich high density lipoproteins species accumulate in the plasmas of diettreated animals $(1,2)$. The responses in man, although qualitatively similar, are muted (1). In the rat, a high cholesterol diet alone produces modest changes, but the combination of dietary cholesterol and hypothyroidism produces severalfold increases in plasma cholesterol, as well as rises in $\beta$-VLDL, intermediate density lipoproteins, and apo $\mathrm{E}$ high density lipoproteins. Concomitantly, apo B and E levels increase in plasma by $\sim 50 \%$, whereas apo AI levels remain relatively constant $(1,2)$. The diet-induced changes in plasma reflect, at least in part, altered rates of secretion into the plasma and

Address reprint requests to Dr. David Alpers, Gastroenterology Division, Division of Medicine, Washington University School of Medicine; 660 South Euclid, Box 8124, St. Louis, MO 63110. Dr. Sussman's present address is Baylor College of Medicine, Houston, TX 77030; Dr. Go's present address is University of Alabama, Birmingham, AL 35924.

Received for publication 12 November 1986 and in revised form 10 November 1987.

J. Clin. Invest.

(c) The American Society for Clinical Investigation, Inc.

$0021-9738 / 88 / 05 / 1615 / 06 \$ 2.00$

Volume 81, May 1988, 1615-1620 intestinal lymph of lipoproteins by hepatocytes and enterocytes, respectively (3). There are changes, not only in the amounts, but also in the compositions of the lipoproteins that are secreted. For example, nascent VLDL isolated from the Golgi of enterocytes of cholesterol-fed rats are enriched in their contents of cholesterol and apo AIV (4), whereas the nascent VLDL isolated from the Golgi of hepatocytes are enriched in apo E (5). Apo AI content does not appear to be remarkably altered in the nascent VLDL isolated from either organ. Altered production, however, does not totally account for the changes in plasma, because the high cholesterol diets and hypothyroidism also produce marked effects on the interconversion and clearance of particles from plasma. The effects of these diets on apoprotein synthesis and on the levels at which regulation of synthesis occur thus must be studied directly in the appropriate tissues. Because apo AI, apo AIV, and apo E in plasma appear to be differently affected by manipulations of dietary and thyroid status, we chose to study the regulation and synthesis of these apoproteins.

\section{Methods}

\section{Animals and diets}

200-250-g male Sprague-Dawley rats (Chappell Breeders, St. Louis, MO) were fed diets obtained from ICN Nutritional Biochemicals, Cleveland, $\mathrm{OH}$. The contents of the diets were as follows: diet 1, Wayne Lab Blox rat chow (Continental Grain Co., Chicago, IL) containing $24.5 \%$ protein, $4.3 \%$ vegetable fat, $7.8 \%$ ash, $13.3 \% \mathrm{H}_{2} \mathrm{O}, 3.7 \%$ fiber, and $47.7 \%$ nitrogen-free carbohydrate (of which $5 \%$ was monoor disaccharides) plus $5 \%$ lard and $0.3 \% \mathrm{Na}$ taurocholate; diet 2, diet 1 plus $2 \%$ cholesterol; diet 3 , diet 1 plus $0.1 \%$ propylthiouracil (PTU) and diet 4 , diet 2 plus $0.1 \%$ PTU. Diet 1 is the proper control diet for the other diets, as its contents, including $5 \%$ added lard, are present in diets 2-4. Experiments were also performed using rat chow without added lard, and these results are included in the text for comparison with diet 1 . Animals were killed at 2-3 wk, after 3-4 h of fasting. Plasma lipid and apoprotein concentrations were measured by enzymatic assays and RIA $(6,7)$. Apoproteins were also measured in tissues, hepatocytes, or intestinal organ explants. All assays reported were performed in duplicate; $n$ refers to the number of animals from which the tissues were taken.

\section{mRNA levels}

Cell-free translation. Total RNA was extracted from liver and jejunum with guanidine $\mathrm{HCl}$ according to Gordon et al. (8). Untoasted wheat germ was a gift from General Mills (Vallejo, CA) and a lysate was prepared according to the method of Roberts and Paterson (9). $\left[{ }^{35} \mathrm{~S}\right]-$ Methionine of the highest specific activity was purchased from Amersham Corp. (Arlington Heights, IL) or New England Nuclear (Boston, MA). In vitro translation was carried out with conditions as described (10), with the addition of $1,200 \mathrm{U} / \mathrm{ml}$ of placental RNase inhibitor (RNAsin; Promega Biotec, Madison, WI) and $300 \mu \mathrm{g}$ total RNA/ml.

1. Abbreviations used in this paper: GMP, guanosine monophosphate; PTU, propylthiouracil; SSC, sodium chloride sodium citrate buffer. 
Monospecific antibodies against apo AI and apo AIV were incubated overnight at $4^{\circ} \mathrm{C}$ and precipitated apoproteins were separated on SDS gels. The appropriate bands were exercised and radioactivity was eluted from the gels in $1.25 \%$ SDS overnight and then counted using 3 a70 scintillation cocktail. These counts were expressed as a fraction of the total counts that were TCA precipitable. All determinations were performed on three separate incubations for each experimental point.

Northern blot. Since the cDNA probes available to us did not permit stringent washing conditions, we quantitated mRNA-cDNA hybrids by Northern blot rather than by dot blots, to avoid any background contamination. Northern blots were made using a $1.5 \%$ agarose gel and total RNA extracted as above. The blots were probed with a ${ }^{32} \mathrm{P}$-end labeled 23-oligomer (5'CTACTTCACCCAGCTAAGCAACA) to apo AIV synthesized by the Protein Sequencing Facility of Washington University Medical Center. A nick-translated clone pAI-113 to human apo AI was obtained from Dr. J. L. Breslow, The Rockefeller University, New York (11). Hybridizations were carried out at $23^{\circ} \mathrm{C}$ in $0.2 \% \mathrm{SDS} / 1.0 \times$ sodium chloride sodium citrate buffer (SSC) and $37^{\circ} \mathrm{C}$ in $0.2 \%$ SDS $/ 0.1 \times \mathrm{SSC}$, respectively (8). Filters were washed at $23^{\circ} \mathrm{C}$ with $1 \times \mathrm{SSC} / 0.2 \%$ SDS and $0.1 \times \mathrm{SSC} / 0.2 \% \mathrm{SDS}$, respectively. Relative quantities of the hybridized heteroduplexes of the appropriate size (1.2 kb for apo AI, $1.8 \mathrm{~kb}$ for apo AIV) were determined by laser densitometry (Biomed Instruments, Inc., Fullerton, CA) of preflashed XAR-5 film.

\section{Apoprotein synthesis and secretion}

Organ explants. Incorporation of labeled amino acid was used to assess synthesis in intestinal explants, because the significant plasma contamination of organ explants prevented the use of RIA to measure total apoprotein content as a function of synthesis, as was done using hepatocyte cultures. Thirty 2-mm full-thickness punch biopsies of the jejunum were incubated with $50 \mu \mathrm{Ci} / \mathrm{ml}\left[{ }^{35} \mathrm{~S}\right]$ methionine in $1 \mathrm{ml} \mathrm{DME}$ medium supplemented with $40 \mathrm{mM}$ sodium bicarbonate, $24 \mathrm{mM}$ galactose, and essential amino acids minus methionine. Incubations were carried out at $37^{\circ} \mathrm{C}$ in an atmosphere of $95 \% \mathrm{O}_{2}$ and $5 \% \mathrm{CO}_{2}$ for $4 \mathrm{~h}$ (12). The tissue was homogenized in PBS with $1 \%$ Triton, $0.1 \mathrm{M}$ benzamidine, and $0.03 \mathrm{M}$ PMSF. Both the tissue and medium were immunoprecipitated overnight with the monospecific apo AI and apo AIV antibodies. Proteins were separated in $10 \%$ polyacrylamide gel with $0.1 \%$ SDS according to Laemmli (13). The bands corresponding to the apoproteins were identified by fluorography and excised, and the radioactivity was eluted in $1.25 \%$ SDS and counted in a xylene-based scintillating mixture (3a70). Total protein synthesis was assessed by boiling an aliquot in 10\% TCA and counting in the 3a70 cocktail.

Primary hepatocyte cultures. Primary hepatocyte cultures were established in modified DME medium. After 6 and $24 \mathrm{~h}$, the cells and media were assayed for apo E by RIA $(6,7)$. Since apo E production was linear over $24 \mathrm{~h}$, the total apoprotein content in both the cells and medium was assessed at both times and the values combined after converting the content to nanograms per milligram protein per $24 \mathrm{~h}$ in culture.

Polysome extraction. Polysomes were extracted from both the liver and jejunum of diet-treated rats according to the method of Strauss et al. (14). This method entails removal of the polysomes from the 2.5- to the 1.0-M sucrose interface, dilution with $50 \mathrm{mM}$ Tris- $\mathrm{HCl}, \mathrm{pH} 7.4,25$ $\mathrm{mM} \mathrm{NaCl}, 5 \mathrm{mM} \mathrm{MgCl}$, and $200 \mu \mathrm{g} / \mathrm{ml}$ sodium heparin, and centrifugation at $65,000 \mathrm{rpm}$ for $1.5 \mathrm{~h}$. Pellets were resuspended at a concentration of 50-200 $A_{260} \mathrm{U} / \mathrm{ml}$ and stored in liquid $\mathrm{N}_{2}$ for future use. Liver cell sap $(105,000 \mathrm{~g}$ supernatant) was added to the jejunal mucosa to inhibit ribonuclease activity. Since it was possible that apolipoprotein contamination might occur from the added liver cell sap, this same fraction was added to rat antral tissue (which does not produce apoproteins) and polysomes were isolated. No apo AI or AIV could be detected in the polysomal pellet. Translation of in vivo-initiated nascent polypeptide chains was carried out in the presence of $1.5 \mathrm{mM}$ 7-guanosine monophosphate (7-GMP) to inhibit the in vitro initiation of new polypeptide chains from the polysome complex (15). Polysomal runoff thus assessed the rate of in vivo initiation of specific peptides. In these experiments, 7-GMP inhibited total polysomal translation by $10-15 \%$, and shortened the time for apoprotein content to peak in vitro from 45 to $30 \mathrm{~min}$. Samples were standardized by adding $0.3 \mathrm{~A}_{260}$ units (15 $\mu \mathrm{g}$ of RNA) to each translation reaction. Translations were carried out for $60 \mathrm{~min}$, since no further increase in apoprotein content was noted after $30 \mathrm{~min}$. Immunoprecipitation was carried out as described above.

Since the purpose of the assay was to assess the number of polysomes (encoding a specific peptide) that were initiated in vivo, we measured the apoprotein content of the polysome by Western blotting after the runoff period in vitro. This approach is consistent with the use of the runoff assay as originally described (14). This assay does not determine the rate of synthesis, but rather reflects the amount of protein that was translated in vivo at the time of the polyribosome isolation. The rate of in vitro translation (or completion) is rapid and not an independent determinant, as all factors necessary for completion of the chains are added.

To be certain that already completed peptide chains were not included in the runoff assay, we performed a mixing experiment, adding liver cell sap as a source of completed (liberated) apoproteins to the liver or intestinal polysomes. Apoprotein AI content of liver cell sap was titrated by Western blot analysis so that an amount of reactive peptide equal in amount to that found at the end of a polysome runoff experiment was added. The source of polysomes was fat-fed jejunum and liver. After mixing, polysomes were reisolated from the sucrose interface, precipitated by heparin, and runoff analyses were performed. The amount of apo AI recovered from the intestinal and liver polysomes after mixing with the liver cell sap was 87 and $82 \%$ of the value obtained after runoff of primarily isolated polysomes. The slightly lower values obtained after mixing might be due to loss of bound, uncompleted peptide during reisolation. No evidence was found, however, for trapping or contamination of completed apo AI in the polysome pellet. These experiments were repeated for the cholesterol/PTU fed animals, with recoveries of 92 and $83 \%$ for intestinal and liver polysomes, respectively. In the absence of evidence for polysomal contamination by apoproteins, Western blot analysis of total apolipoprotein content at the end of the runoff was used as a measure of total peptide chains initiated in vivo.

The apo AI and apo AIV produced by runoff of polysomes in $25-\mu 1$ translation volumes were separated by SDS-PAGE, followed by overnight transfer to nitrocellulose filters. The blots were probed using nonlabeled monospecific AI and AIV antibodies and ${ }^{125}$ I-protein A, according to the method of Burnette (16). Protein A was obtained from Sigma Chemical Co., St. Louis, $\mathrm{MO}$ and ${ }^{125} \mathrm{I}$ from Industrial Nuclear, St. Louis, MO. Relative quantities of complexes of the two apoproteins from the runoff assays were determined by laser densitometry scanning as for the Northern blots. All assays were performed in triplicate.

\section{Results}

Concentration of lipids and apoproteins in plasma (Table I). As reported by us and others previously, cholesterol feeding had modest effects on plasma triglycerides levels (17), whereas PTU decreased triglycerides $(18,19)$. Cholesterol feeding alone approximately doubled plasma cholesterol concentrations. With the diet combination of cholesterol plus PTU, cholesterol concentrations increased four- to fivefold. None of the treatments significantly affected apo AI levels. PTU treatment with or without the addition of cholesterol, however, increased plasma apo E concentrations.

Apoprotein synthesis and secretion. Organ explants from the jejunum of rats fed the various diets were incubated in medium with $\left[{ }^{35}\right.$ S $]$ methionine. After $4 \mathrm{~h}$, tissues and media were assayed for apo AI and apo AIV by immunoprecipitation with the individual antisera. Significantly increased amounts of newly synthesized apo AI and apo AIV were secreted into 
Table I. Effects of High Cholesterol and Hypothyroidismproducing Diets on Plasma Lipid and Apoprotein Levels

\begin{tabular}{lcccc}
\hline \multicolumn{1}{c}{ Diet } & Triglycerides & Cholesterol & Apo AI & Apo E \\
\hline \multicolumn{3}{c}{$m g / d l$} \\
Fat & $56 \pm 21$ & $65 \pm 4$ & $42 \pm 6$ & $26 \pm 4$ \\
Cholesterol & $84 \pm 36$ & $118 \pm 42^{*}$ & $47 \pm 5$ & $20 \pm 6$ \\
PTU & $27 \pm 4^{*}$ & $88 \pm 4^{*}$ & $41 \pm 7$ & $31 \pm 4^{*}$ \\
Chol \& PTU & $59 \pm 40$ & $293 \pm 73^{*}$ & $46 \pm 15$ & $35 \pm 1^{*}$ \\
& & & & $m g / d l$ \\
\hline
\end{tabular}

$n=3$ animals, results are means \pm SD. Abbreviation: Chol, cholesterol. ${ }^{*} P<0.03$, compared with the fat diet.

the medium from explants of animals on the cholesterol/PTU diet. PTU alone decreased secretion, whereas cholesterol alone had no effect (Table II). Levels of these apoproteins in the tissue itself were not significantly different from control levels ( $0.026 \%$ for apo $\mathrm{AI}$ and $0.033 \%$ for apo AIV), implying that the newly synthesized proteins are efficiently secreted. Apo E secretion was not measured in intestinal explants because apo $E$ is not apparently secreted from enterocytes into lymph.

The cultured hepatocytes of the same animals showed increased synthesis of apo AI on the cholesterol-only diet, and no significant change on the PTU/cholesterol diet, whereas PTU alone depressed synthesis (Table III). Intestinal and liver apo AI thus responded differently to the diets. The hepatocyte synthesis of apo E on cholesterol and cholesterol/PTU diets rose comparably, whereas PTU alone did not alter synthesis. Tissue concentrations of apo AI and apo $\mathrm{E}$ on the fat diets were 11.9 and $445 \mathrm{ng} / \mathrm{mg}$ protein, respectively. These concentrations were not much changed with experimental manipulations, so that most of the changes in synthesis (Table III) were due to altered secretion into the medium.

Concentration of mRNAs encoding apoproteins. Our preliminary work had suggested that there might be little change in mRNA concentration of apo AI with fat feeding (20). Constant amounts of total RNA from liver and jejunum from rats fed the experimental diets were translated in the in vitro wheat germ lysate system. The mean percent translation of mRNA in

Table II. Production of Apoproteins in Rat Intestinal Organ Culture

\begin{tabular}{llc}
\hline \multicolumn{1}{c}{ Condition } & \multicolumn{1}{c}{ Apo AI } & Apo AIV \\
\hline \multicolumn{3}{c}{ \% of total counts } \\
Fat & $0.022 \pm 0.011$ & 0.025 \\
Chol & $0.029 \pm 0.01$ & $0.025 \pm 0.006$ \\
PTU & $0.006 \pm 0.002^{*}$ & $0.008 \pm 0.004^{\ddagger}$ \\
Chol/PTU & $0.030 \pm 0.006^{*}$ & $0.037 \pm 0.002^{*}$ \\
\hline
\end{tabular}

Apoprotein secretion was assessed in the medium of organ explants by immunoprecipitation and electrophoretic isolation, and expressed as percent of total protein synthesis, as described in Methods. All experiments represent the mean \pm 1 SD of duplicate explant incubations from six separate animals. A two-tailed $t$ test was used to compare each group with the fat-fed control.

$* P<0.05$.

${ }^{\ddagger} P<0.01$. The mean percent of counts in three animals fed regular rat chow was 0.020 for apo $\mathrm{AI}$ and 0.022 for apo AIV.
Table III. Production of Apoproteins in Rat Hepatocyte Cultures

\begin{tabular}{lcc}
\hline \multicolumn{1}{c}{ Condition } & Apo AI & Apo E \\
\hline \multicolumn{3}{c}{ ng/mg protein } \\
Fat & $46.4 \pm 4.5$ & $1,451.0 \pm 176$ \\
Cholesterol & $104.9 \pm 10.2^{*}$ & $2,479.0 \pm 364^{*}$ \\
PTU & $28.0 \pm 3.1^{*}$ & $1,619.0 \pm 138$ \\
Chol/PTU & $60.0 \pm 4.3$ & $2,341.0 \pm 248^{*}$
\end{tabular}

Apoprotein content was determined by RIA, as described in Methods. The total content for each diet was obtained by adding the tissue content and the amount secreted into the medium in $24 \mathrm{~h}$. All numbers refer to the mean \pm 1 SEM. for duplicate primary cultures from six separate animals. Statistical analysis was performed as in Table II.

$* P<0.05$

liver of three animals fed regular rat chow was $102 \%$ for apo AI and $95 \%$ for apo AIV when compared with the fat diet; in the intestine, comparable values were 78 and $82 \%$, respectively. These data confirm the earlier studies that showed no significant increase in mRNA concentration after fat feeding (21). In RNA extracted from the jejunum of rats fed experimental diets, mRNA concentrations encoding both apo AI and apo AIV were not increased compared with the fat control diet (Table IV). In fact, the content from the cholesterol/PTU animals was significantly decreased. Apo AIV mRNA levels were unchanged in liver, but apo AI mRNA levels were decreased by hypothyroidism (Table IV). By contrast, apo E mRNA content in liver rose on the cholesterol/PTU diet (Table IV), paralleling the hepatic secretory rate after that diet (Table III).

To substantiate that the translation assays accurately reflected mRNA levels, apo AI mRNA concentrations in intestine were also assayed by Northern blotting. $10 \mu \mathrm{g}$ of RNA from liver and jejunum of each dietary group were used for Northern blot analysis. Quantitative densitometry of these gels showed differences in radioactivity that closely paralleled the translational assessments in all conditions except one (liver in PTU diet), and confirmed the lack of rise in apo AI mRNA content in either organ and in all diets (Table V).

Table IV. mRNA Content Assessed by Cell-free Translation

\begin{tabular}{lcccc}
\hline Condition & Organ & Apo I & Apo AIV & Apo E \\
\hline & & & \% translatable $m R N A$ \\
Fat & Liver & $0.14 \pm 0.01$ & $0.12 \pm 0.02$ & $0.51 \pm 0.043$ \\
Cholesterol & & $0.085 \pm 0.008^{*}$ & $0.11 \pm 0.014$ & $0.6 \pm 0.031$ \\
PTU & & $0.042 \pm 0.009^{*}$ & $0.09 \pm 0.012$ & $0.3 \pm 0.023^{*}$ \\
Chol/PTU & & $0.10 \pm 0.009^{*}$ & $0.11 \pm 0.07$ & $1.2 \pm 0.023^{*}$ \\
Fat & Intestine & $1.51 \pm 0.21$ & $1.014 \pm 0.02$ & \\
Cholesterol & & $1.271 \pm 0.17$ & $0.72 \pm 0.12$ & \\
PTU & & $1.19 \pm 0.23$ & $0.80 \pm 0.02$ & \\
Chol/PTU & & $0.83 \pm 0.15^{*}$ & $0.46 \pm 0.03^{*}$ &
\end{tabular}

mRNA content was determined by translational analysis and recorded as the percent of total translatable mRNA, as described in Methods. Data are reported as the mean \pm 1 SD for duplicate translations from six separate animals. Statistical analysis was performed as in Table II.

$* P<0.05$. The mean percent translatable mRNA in liver of three animals fed regular rat chow was 0.14 for apo $\mathrm{AI}$ and 0.11 for apo AIV; corresponding means for intestinal mRNA were 1.21 and 0.82 . 
Table V. Comparison of apo AI mRNA Content by Translational and Blot Analysis

\begin{tabular}{llllll}
\hline & \multicolumn{2}{c}{ Liver } & & \multicolumn{2}{c}{ Jejunum } \\
\cline { 2 - 3 } \cline { 5 - 6 } Condition & Translation & Blot & & Translation & Blot \\
\hline Fat & 1.0 & 1.0 & & 1.0 & 1.0 \\
Cholesterol & 0.61 & 0.8 & & 0.84 & 1.0 \\
PTU & 0.3 & 0.75 & & 0.79 & 0.9 \\
Chol/PTU & 0.7 & 0.5 & & 0.55 & 0.5
\end{tabular}

The data for translational analysis are taken from the mean values of Table IV. The Northern blot analysis is derived from the mean of three separate Northern blots, scanned by laser densitometry. RNA from individual animals was used for each blot. In each case, the mean values of the fat-fed control diet were given the value of 1.0, and the other mean values were related to the control.

Polysome runoff synthesis of apoproteins. Although the PTU and cholesterol/PTU regimens altered the jejunal production of apo AI and apo AIV, mRNA concentrations were unchanged in the intestine, raising the possibility that posttranscriptional (i.e., translational) factors could have been operating. A direct assay for translational events initiated in vivo is the polysome runoff analysis, in which completion of synthesis is measured per unit of polysomal RNA. To eliminate the possibility of in vitro peptide initiation, the analogue 7-GMP was added to the translation mix. The apoproteins synthesized were identified by Western blotting and quantified by scanning densitometry. Significant increases in in vitro apo AI and apo AIV production were seen in jejunum on the cholesterol/PTU diet, but not on the other regimens (Fig. 1 and Table VI). No significant differences were noted in the production of apo AI or apo AIV by hepatic polysomes on any of the diets compared with the control fat diet (Table VI).

The results of polysome runoff in rats fed a standard chow diet revealed values for apo AI that were $35 \%$ (jejunum, see Fig. 1, lane $A$, left) and $28 \%$ (liver) of the control fat diet; corresponding values for apo AIV runoff were $72 \%$ (see Fig. 1, lane $A, r i g h t$ ) and $68 \%$. Thus, fat feeding alone stimulates apo $\mathrm{AI}$ and, to a lesser extent, AIV synthesis by translational control. These results confirm earlier suggestions of the level of

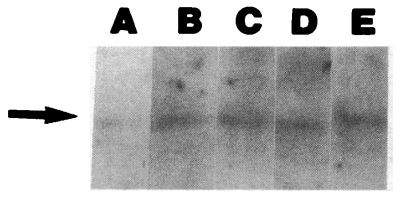

apo A-I

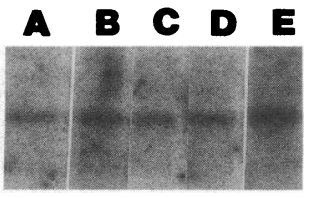

apo $A-\mathbb{Z}$
Figure 1. Western blot of translation elongation products of intestinal polysomes isolated from rats that were fed different diets. Polysomes were isolated from rat jejunum as described in Methods. 7-GMP was used to block de novo initiation. Proteins synthesized from these polysomes were electrophoresed and transferred as described previously. The in vivo initiated apo $\mathrm{AI}$ and apo AIV proteins were identified by monospecific antibodies on Western blots as described in Methods. Lane $A$, chow-fed control diet; $B$, fat (lard) diet; $C$, cholesterol diet; $D$, PTU diet; and $E$, cholesterol/PTU diet. Only the portion of the blots with an immunoprecipitated band are shown.
Table VI. Polysome Runoff Synthesis of Hepatic and Jejunal Apoproteins

\begin{tabular}{lcc}
\hline \multicolumn{1}{c}{ Condition } & Apo AI & Apo AIV \\
\hline Jejunum & & \\
Fat & $81.76 \pm 7.64$ & $33.33 \pm 5.77$ \\
Cholesterol & $46.67 \pm 5.77^{*}$ & $36.67 \pm 11.55$ \\
PTU & $88.33 \pm 22.55$ & $46.67 \pm 10.77$ \\
Chol/PTU & $115.00 \pm 18.03^{*}$ & $90.00 \pm 17.32^{*}$ \\
Liver & & \\
Fat & $116.67 \pm 5.77$ & $30.00 \pm 10.00$ \\
Cholesterol & $126.67 \pm 15.33$ & $36.67 \pm 5.77$ \\
PTU & $120.00 \pm 36.06$ & $40.00 \pm 34.64$ \\
Chol/PTU & $120.00 \pm 10.00$ & $36.67 \pm 15.25$ \\
\hline
\end{tabular}

Data are expressed in arbitrary units derived by laser densitometry scanning of Western blots. Comparable amounts of jejunal and hepatic polysomes from each diet were run off as described in Methods. Each point is the mean \pm 1 SD based on three separate observations from individual animals. A two-tailed $t$ test was used to compare each experimental group with the fat-fed control.

$* P<0.05$.

regulation, based on unchanging mRNA content (21). Moreover, this finding is consistent with the interpretation that polysome runoff assesses the degree of polysome initiation in vivo.

\section{Discussion}

In confirmation of previous findings (22), apo $\mathrm{E}$ levels rose on both of the PTU-containing diets, as well as on cholesterol alone. The hepatic synthesis of apo E did not change on PTU feeding alone. The rise in plasma levels on this regimen thus must have been due to delayed clearance. Hypothyroidism is known to delay the clearance of apo E-containing lipoproteins from plasma, probably because of decreased activity of LDL (apo B, E) receptors on a variety of cells. Hepatic synthesis of apo $\mathrm{E}$ was increased by cholesterol feeding and by the cholesterol/PTU combination. The increased synthesis plus the PTU-induced delayed clearance probably explain the rise in plasma levels on the combined diet. Hepatic levels of apo $\mathrm{E}$ mRNA also rose on the cholesterol/PTU diet, suggesting that on this experimental regimen, apo E synthesis was regulated at the pretranslational level, in agreement with Lin-Lee et al. (23).

The production of hypothyroidism by PTU was also associated with transcriptionally regulated alterations in apoprotein synthesis (Table VII). Apo AI synthesis and mRNA content in liver and intestine and apo AIV synthesis and mRNA content in intestine fell comparably, without any change in polysome runoff synthesis. Although we did not study apo AIV synthesis in the liver, the responses of the two tissues were remarkably similar for apo AI. The hepatic mRNA content for apo $\mathrm{E}$ fell in hypothyroid animals (Table IV), but the synthesis rate did not (Table III). Except for apo E synthetic rate, the response to hypothyroidism was a generalized depression of apoprotein mRNA content and synthesis.

The liver and intestine differed greatly, however, in their response to the atherogenic diet (cholesterol/PTU). Whereas 
Table VII. Summary of Apoprotein Responses to Fat Feeding

\begin{tabular}{|c|c|c|c|c|c|c|}
\hline Proposed level of regulation & Apoprotein & Tissue & Stimulus & Synthesis & mRNA content & Polysome runoff \\
\hline & & & & & \% of control & \\
\hline \multirow[t]{4}{*}{ Pretranslational } & $\mathbf{E}$ & $\mathbf{L}$ & Chol/PTU & 161 & 250 & ND \\
\hline & AI & I & PTU & 60 & 30 & NC \\
\hline & AI & I & PTU & 30 & 30 & NC \\
\hline & AIV & I & PTU & 38 & 72 & NC \\
\hline \multirow[t]{2}{*}{ Translational/initiation in vivo } & AI & I & Chol/PTU & 136 & 70 & 145 \\
\hline & AIV & I & Chol/PTU & 150 & 45 & 280 \\
\hline \multirow[t]{3}{*}{ Translational/mechanism unknown } & $\mathbf{E}$ & $\mathbf{L}$ & Chol & 171 & NC & ND \\
\hline & AI & $\mathbf{L}$ & Chol & 228 & 62 & ND \\
\hline & AI & $\mathbf{L}$ & Chol/PTU & 129 & 71 & $\mathrm{NC}$ \\
\hline
\end{tabular}

Regulation was considered pretranslational if synthesis and mRNA content changed in parallel (with no change in polysome runoff), and translational if changes in synthesis were in the opposite direction to changes in mRNA content. Translational regulation was considered to occur at the rate of initiation of synthesis in vivo if the altered synthesis was accompanied by parallel changes in polysome runoff synthesis. NC, no change.

hepatic apo E synthesis increased by pretranslational regulation (see above), the mechanism for altered synthesis of apo AI and apo AIV in intestine was translational (Table VII). In both instances (apo AI and apo AIV), synthesis was increased while mRNA content was falling. Polysome runoff (i.e., completion of chains already initiated in vivo), was increased in both cases. The protein-synthesizing system used in these experiments allows completion of nascent peptide chains bound to polyribosomes. Because these chains were initiated in vivo, apoprotein content reflects the amount of specific protein that was being translated in vivo at the time of polysome isolation.

These data are consistent with our earlier observations, in which apo AI mRNA concentration remained unchanged over a 4-h period (21). In contrast, acute polyunsaturated triacylglycerol feeding has been shown in rat to produce an increase in apo AI (24) and apo AIV (25) levels by translational analysis, or in apo AIV mRNA by Northern blotting (20). These studies used RNA from the entire intestine. We extracted RNA from the jejunum only, since apo AI synthesis may be regulated independently in the jejunum and ileum. In addition, we have assessed the response to chronic saturated fat feeding. One other study has studied the intestinal apoprotein AI response after chronic feeding of an atherogenic diet (26), and found no change in apo AI synthesis, unlike the present study. The diet used by those authors differed both in the percent cholesterol (1\% vs. $2 \%$ ) and in the basic chow (Purina vs. ICN), and the responses were clearly different. Davidson et al. produced a serum cholesterol level two times higher, found no decline in triglycerides on the PTU diet, and reported a 50\% decline in serum apo AI levels on the cholesterol/PTU diet (26), all findings that differ from ours (Table I).

The liver also differed from the intestine in its response to cholesterol-containing diets (Table VII). Both apo E and apo AI synthesis increased without a parallel increase in mRNA content, consistent with regulation at the level of translation. However, where polysome runoff synthesis was examined (apo AI), it did not change. This was different from the response of intestine to the atherogenic diet. It is not clear why the increased synthesis was not reflected in enhanced polysome runoff, especially since the assay seems to reflect the differences in synthetic rate between regular chow and fat-supplemented diets. The interesting fact remains, however, that in response to cholesterol-containing diets, the liver and intestine seem to alter apoprotein synthesis by different mechanisms in response to the same stimuli. Whereas the liver responds to cholesterol feeding by increasing apo AI and apo E synthesis about twofold, intestinal synthesis of apo AI and AIV was not changed (Table II). Although the details of the response differ from ours, Apostolopoulos et al. found that rat intestinal and liver mRNA levels changed in nonparallel fashion in response to a similar feeding experiment (27).

Studies of hormonal systems indicate that many proteins are under pretranslational control, in which increased protein synthesis is mediated through increased mRNA levels (28-29). Translational control, however, in which protein synthesis is increased by more efficient translation of unchanged amounts of mRNA, is also an important regulatory mechanism (30). Both mechanisms may be involved for a single protein. For example, dibutyryl cAMP induced tyrosine aminotransferase synthesis in rat liver by a transient mRNA elevation and then by a second delayed, but sustained, elevation in translation of a basal level of mRNA (31). Our studies show that apo AI synthesis is increased by a number of different dietary conditions, while its mRNA concentration remains constant. This dissociation of apo AI mRNA and protein synthesis indicates that translational control, rather than pretranslational regulation, is the important control mechanism in apo AI levels in chronically fat-fed rats.

Clearly, there is no single pattern of regulation of apoprotein synthesis or secretion after fat feeding. The rates and mechanisms of altered synthesis seem to vary according to the type of lipid fed (triglyceride vs. cholesterol), type of apoprotein studied, and tissue of origin. The wide variety of possible patterns will become clear as more experimental situations are explored. These may provide clues to the actual signals whereby dietary manipulations influence apoprotein synthesis and secretion in the intestine and liver.

\section{Acknowledgments}

The authors wish to thank Grace Kennedy and Joyce Rudolph for typing the manuscript.

This work was supported by National Institutes of Health (NIH) grants HL-15427 and DK-14038, and NIH training grant AM-07130. 


\section{References}

1. Mahley, R. W., T. L. Innerarity, S. C. Rall, Jr., and K. H. Weisgraber. 1984. Plasma lipoproteins: apolipoprotein structure and function. J. Lipid Res. 25:1277-1294.

2. Cole, T. G., I. Kuisk, W. Patsch, and G. Schonfeld. 1984. Effects of high cholesterol diets on rat plasma lipoproteins and lipoprotein-cell interactions. J. Lipid Res. 25:593-603.

3. Windmueller, H. G., and A. E. Spaeth. 1972. Fat transport and lymph and plasma lipoprotein biosynthesis by isolated intestine. $J$. Lipid Res. 13:92-105.

4. Swift, L. L., P. D. Soule, M. E. Gray, and V. S. LeQuire. 1984. Intestinal lipoprotein synthesis. Comparison of nascent Golgi lipoproteins from chow-fed and hypercholesterolemic rats. J. Lipid Res. 25:1-13.

5. Howell, K. E., and G. E. Palade. 1982. Heterogeneity of lipoprotein particles in hepatic Golgi fraction. J. Cell Biol. 92:833-845.

6. Schonfeld, G., M. S. Fricke, and A. P. Bailey. 1976. Measurement of apolipoprotein $\mathrm{AI}$ in rat high density lipoprotein and in rat plasma by radioimmunoassay. J. Lipid Res. 17:25-29.

7. Patsch, W., and G. Schonfeld. 1983. Role of insulin in lipoprotein secretion by cultured rat hepatocytes. J. Clin. Invest. 71:11611174.

8. Gordon, J. I., D. P. Smith, R. Andy, D. H. Alpers, G. Schonfeld and A. W. Strauss. 1982. The primary translation product of rat intestinal apolipoprotein A-I mRNA is an unusual preproprotein. J. Biol. Chem. 257:971-978.

9. Roberts, B. E., and B. M. Paterson. 1973. Efficient translation of tobacco mosaic virus RNA and rabbit globin 9s RNA in a cell-free system from commercial wheat germ. Proc. Natl. Acad. Sci. USA. 70:2330-2334

10. Sussman, N. L., S. Seetharam, M. C. Blaufuss, and D. H. Alpers. 1986. Translation of rat intestinal RNA yields two alkaline phosphatases. Biochem. J. 234:563-568.

11. Breslow, J. L., D. Ross, J. McPherson, H. Williams, D. Kurnit, A. L. Nussbaum, S. K. Karathanasis, and V. I. Zannis. 1982. Isolation and characterization of cDNA clones for human apolipoprotein A-I. Proc. Natl. Acad. Sci. USA. 79:6861-6865.

12. Browning, T. H., and J. S. Trier. 1969. Organ culture of mucosal biopsies of human small intestine. J. Clin. Invest. 48:1423-1432.

13. Laemmli, U. K. 1970. Cleavage of structural proteins during the assembly of the head of bacteriophage T4. Nature (Lond.). 227:680-685.

14. Strauss, A. W., A. W. Alberts, S. Hennessy, and P. R. Vagelos. 1975. Regulation of synthesis of hepatic fatty acid synthetase: polysomal translation in a cell-free system. Proc. Natl. Acad. Sci. USA. 72:4366-4370.

15. Hickey, E. D., L. A. Weber, and B. Baglioni. 1976. Inhibition of initiation of protein synthesis by 7-methylguanosine-5'-monophosphate. Proc. Natl. Acad. Sci. USA. 73:19-23.
16. Burnette, W. N. 1981. "Western blotting": electrophoretic transfer of proteins from sodium dodecyl sulfate-polyacrylamide gels to unmodified nitrocellulose and radiographic detection with antibody and radioiodinated protein A. Anal. Biochem. 112:195-203.

17. Delamatre, J. G., and P. S. Roheim. 1983. The response of apolipoprotein A-IV to cholesterol feeding in rats. Biochim. Biophys. Acta. 751:210-217.

18. Dory, L., and P. S. Roheim. 1981. Rat plasma lipoproteins and apolipoproteins in experimental hypothyroidism. J. Lipid Res. 22:287-296.

19. Dolphin, P. J., and S. J. Forsyth. 1983. Nascent hepatic lipoproteins in hypothyroid rats. J. Lipid Res. 24:541-551.

20. Gordon, J. I., D. P. Smith, D. H. Alpers, and A. W. Strauss. 1982. Proteolytic processing of the primary translation product of rat intestinal apolipoprotein A-IV mRNA. J. Biol. Chem. 257:8418-8423.

21. Blaufuss, M. C., J. I. Gordon, G. Schonfeld, A. W. Strauss, and D. H. Alpers. 1984. Biosynthesis of apolipoprotein C-III in rat liver and small intestinal mucosa. J. Biol. Chem. 259:2452-2456.

22. Krul, E. S., and P. J. Dolphin. 1982. Secretion of nascent lipoproteins by isolated hepatocytes from hypothyroid and hypothyroid, hypercholesterolemic rats. Biochim. Biophys. Acta. 713:609-621.

23. Lin-Lee, Y. C., Y. Tanaka, C. T. Lin, and L. Chan. 1981. Effects of an atherogenic diet on apolipoprotein $\mathrm{E}$ biosynthesis in the rat. Biochemistry. 20:6474-6480.

24. Tanaka, Y., Y. C. Lin-Lee, M. H. Lin-Su, and L. Chan. 1982. Intestinal biosynthesis of apolipoproteins in the rat: apo $\mathrm{E}$ and apo A-I mRNA translation and regulation. Metab. Clin. Exp. 31:861-865.

25. Pessah, M., C. Alvat, N. Amit, and R. Infante. 1985. Isolation and characterization of rat intestinal polyribosomes and RNA during absorption of fat. Increased translation in vitro of Apo A-IV. Biochem. Biophys. Res. Commun. 126:373-381.

26. Davidson, N. O., A. M. Magun, T. A. Brasitus, and R. M. Glickman. 1985. Intestinal apolipoprotein A-1 and B-48 metabolism: effects of sustained alterations in dietary triglyceride and mucosal cholesterol flux. J. Lipid Res. 28:388-402.

27. Apostolopoulos, J. J., G. J. Howlett, and N. Fidge. 1987. Effects of dietary cholesterol and hypothyroidism on rat apolipoprotein mRNA metabolism. J. Lipid Res. 28:642-648.

28. Chan, L., A. R. Means, and B. W. O'Malley. 1978. Steroid hormone regulation of specific gene expression. Vitam. Horm. 36:259-295.

29. Darnell, J. E. 1982. Variety in the level of gene control in eukaryotic cells. Nature (Lond.). 297:365-371.

30. McGuire, D. M., C. D. Olson, H. C. Towle, and M. E. Dempsey. 1984. Translational control of the circadian rhythm of liver sterol carrier protein. J. Biol. Chem. 259:5368-5371.

31. Noguchi, T., M. Diesterhaft, and D. Granner. 1982. Evidence for a dual effect of dibutyryl cyclic AMP on the synthesis of tyrosine aminotransferase in rat liver. J. Biol. Chem. 257:2386-2390. 